\title{
Space buzz heads east
}

\author{
While the Olympics kick off in London, a new international sporting arena is taking shape beyond Earth's \\ orbit. Recent advances in space exploration by China and Japan remind us that curiosity about our \\ universe is a truly universal trait.
}

A new dawn has arrived for space exploration in the Land of the Rising Sun. Over the past year, cinemas in Japan have seen a deluge of films about the Hayabusa mission to the asteroid Itokawa. The spacecraft has been featured in the everpopular Japanese comic books, referenced in a space-themed tokusatsu television show, and has even been made into a Lego toy. Inspired by Hayabusa's successes, at the Asteroids Comets Meteors meeting in Niigata, Japan, this May (http://chiron. mtk.nao.ac.jp/ACM2012/), students from three Japanese schools mingled among the world's leading scientists in smallbodies research.

Two years after its return to Earth, this unmanned space probe continues to pervade Japanese popular culture. Hayabusa, launched by the Japanese Aerospace Exploration Agency (JAXA) in May 2003, was designed to rendezvous with the near-Earth asteroid Itokawa, descend to its surface, kick up some dust, and return a sample to Earth in 2007. The mission was riddled with glitches, but on 13 June 2010, Hayabusa finally became the first spacecraft to return samples from an asteroid. More than 1,500 tiny particles less than 200 micrometres in size were recovered, analysis of which revealed the asteroid's complex formation history, and linked the asteroid family to which Itokawa belongs to ordinary chondrites, the most commonly found meteorites (Science 333, 1113-1131; 2011).

Hayabusa is the first mission to return extraterrestrial surface material since the NASA Apollo and Soviet Luna missions. Its ultimate success after several setbacks provided enough drama to fuel three feature-length films, even though there were no astronauts onboard. The spacecraft experienced a succession of problems with communications and other technical issues that jeopardized the mission. Furthermore, at the asteroid, the sample capture mechanism malfunctioned: until the sample-storage capsule touched down in Australia, it was unclear whether material from the asteroid had been collected at all.

The tribulations of the plucky 'spacecraft that could' - not unlike those of Apollo 13

that eked out a safe return of its crew to Earth after a failed moon landing — are clearly movie material. But the widespread popularity of Hayabusa in Japan echoes the Apollo era in other ways than just as a lesson in near failure. Hayabusa was the first significant Japanese-led space mission and, more than 40 years since millions around the world tuned in to watch Neil Armstrong's first steps on the Moon, is leading a new wave of popular enthusiasm in East Asia for space exploration.

China, too, is making great strides in space exploration with its 20-year-old space programme. China's Shenzou 9 spacecraft with a three-person crew - including

China's first female taikonaut - returned to Earth on 29 June after a 13-day voyage. The mission represented a number of technological achievements, including manually docking with the unmanned orbital module Tiangong-1.

Again harkening back to the Apollo and Luna era, the most momentous events of the mission were broadcast live on China's state television. After earlier setbacks - including the failed 2011 launch of the Phobos-Grunt mission to the Mars system in collaboration with Russia - China is emerging as one of a handful of leading space nations. The latest achievements have undoubtedly led to a spike in the popular interest among the Chinese in science and technology.

China is planning longer and bolder missions, following the success of Shenzou 9
(Science 336, 1630-1637; 2012). JAXA is also preparing for Hayabusa 2, which is scheduled to launch in 2014: another asteroid sample-return mission, this time to the carbonaceous asteroid 1999 JU3. Hayabusa 2 will arrive at the asteroid in 2018 - two years before NASA's competing sample-return mission, OSIRIS-REx (Origins-Spectral Interpretation-Resource Identification-Security-Regolith Explorer), to the carbonaceous asteroid 1999 RQ36. Samples from these target asteroids - which are geochemically more primitive than Itokawa may contain hydrated and carbonaceous minerals. Analysis of such minerals is hoped to provide information about the origin of water and organic materials in the Solar System. Blockbuster space missions have become almost run-off-the-mill
for NASA, but in East Asia, such fame is still a novelty. Whereas the postApollo generation of American children have grown up taking space exploration for granted, Japanese youth are being inspired to really think beyond planet Earth.

NASA's Voyager 1 spacecraft, launched in 1977, is about to reach the edge of our Solar System (see page 518), but planetary exploration is just beginning in Asia. More than 40 years since the first astronauts and cosmonauts left Earth's orbit, the giant leaps for humankind continue only now, the leaps go well beyond Cold War competition as they become a truly international endeavour. 